\title{
Poly(ADP-ribose) polymerase-1 regulates the mechanism of irradiation-induced CNE-2 human nasopharyngeal carcinoma cell autophagy and inhibition of autophagy contributes to the radiation sensitization of $\mathrm{CNE}-2$ cells
}

\author{
ZHI-RUI ZHOU ${ }^{1,2}$, XIAO-DONG ZHU ${ }^{1}$, WEI ZHAO ${ }^{1}$, SONG QU $^{1}$, FANG SU $^{1}$, \\ SHI-TING HUANG ${ }^{1}$, JIA-LING MA ${ }^{1,2}$ and XIAO-YU LI ${ }^{1}$ \\ ${ }^{1}$ Department of Radiation Oncology, Cancer Hospital of Guangxi Medical University and \\ Cancer Institute of Guangxi Zhuang Autonomous Region, Nanning 530021; \\ ${ }^{2}$ Guangxi Medical Scientific Research Center, Guangxi Medical University, Nanning 530021, P.R. China
}

Received December 17, 2012; Accepted March 19, 2013

DOI: $10.3892 /$ or.2013.2382

\begin{abstract}
The aim of the present study was to investigate the role of autophagy in response to ionizing radiation (IR) in CNE-2 human nasopharyngeal carcinoma cells and to demonstrate the function of poly(ADP-ribose) polymerase-1 (PARP-1) in the regulation of IR-induced autophagy. Microtubule-associated protein 1 light chain 3 (LC3) and poly(ADP-ribose) (PAR) were assessed using western blotting. Ultrastructural analysis was performed using transmission electron microscopy (TEM). The percentage of apoptotic cells was assessed by flow cytometry. The MTT method was used to detect cell viability of CNE-2 cells at different time points after IR. Clonogenic survival assays were used to evaluate the radiosensitivity of nasopharyngeal carcinoma cells treated with IR and IR combined with autophagy inhibitor (chloroquine phosphate), with autophagy inducer (rapamycin) or with PARP-1 inhibitor 3-amino benzamide (3AB). IR induced a massive accumulation of autophagosomes detected by TEM and intensified the conversion of cytosolic LC3-I to LC3-II. PARP-1 activation was accompanied by strong upregulation of PAR and LC3-II expression in CNE-2 cells. Compared with radiation alone, chloroquine phosphate (CDP) or $3 \mathrm{AB}$ combined with IR significantly decreased cell viability, as well as the autophagic ratio and LC3-II protein levels. Inhibition of autophagy increased radiation-induced apoptosis; rapamycin (RAPA) significantly decreased cell viability as well, but
\end{abstract}

Correspondence to: Professor Xiao-Dong Zhu, Department of Radiation Oncology, Cancer Hospital of Guangxi Medical University, Nanning 530021, P.R. China

E-mail: xiaodongzhu1966@gmail.com

Key words: autophagy, PARP-1, nasopharyngeal carcinoma, radiosensitivity, chloroquine diphosphate, rapamycin, 3-amino benzamide
RAPA increased the autophagic ratio and LC3-II protein levels; induction of autophagy increased radiation-induced apoptosis. To conclude, PARP-1 regulates IR-induced autophagy, and PARP-1 inhibitor contributes to the radiation sensitization of CNE-2 cells. Blockade of autophagy with CDP enhanced the cytotoxicity of radiotherapy in CNE-2 cells. This suggests that inhibition of autophagy or PARP-1 may be used as an adjuvant therapy to treat nasopharyngeal carcinoma.

\section{Introduction}

Nasopharyngeal carcinoma (NPC), which has the highest incidence in Southeast Asia, remains one of the leading causes of cancer-related mortality in the Cantonese region of Southern China. Radiotherapy is the major treatment for NPC (1). However, radioresistance remains a serious obstacle to successful treatment in many cases $(2,3)$. Thus, an effective way to improve radiation sensitization of nasopharyngeal carcinoma is by identifying the mechanisms involved in NPC radiation resistance. Autophagy is a catabolic process involved in cell growth, development and homeostasis, maintaining a balance between the synthesis, degradation and subsequent recycling of cellular products. The process starts with the formation of the autophagosome or autophagic vacuole. The vacuole membrane then fuses with the lysosomal compartment to deliver the contents into the organelle lumen, where they are degraded and the resulting macromolecules are recycled $(4,5)$. Recent studies have revealed the importance of autophagy in the immune response, inflammatory response, cardiovascular disease, cancer and neurodegenerative disease (6-9). However, the major role that autophagy plays in cancer is controversial (10). Genetic knockout of autophagyrelated genes enhances the development of spontaneous malignancies whereas mice deficient in autophagy-related genes exhibit sensitivity to radiotherapy, chemotherapy or immunotherapy (10-13).

Poly(ADP-ribose) polymerase-1 (PARP-1), activated by DNA strand breaks, participates in the DNA repair process 
physiologically (14). PARP-1 has been implicated in the $\mathrm{G} 2 / \mathrm{M}$ cell cycle checkpoint and has been shown to play an important role in the recovery of DNA damage in in vivo studies. Specifically, PARP-1 has been demonstrated to be an important mediator of DNA base excision repair, which is important in the repair of single-stranded breaks. Moreover, PARP-1 is also known to bind the more lethal doublestranded DNA breaks (15-17). IR induces DNA strand breaks, which mediate its cytotoxic effects. Excessive activation of PARP-1 mediates ionizing radiation (IR)-induced cell death under the status of oxidative stress and DNA damage $(18,19)$. However, it remains elusive whether and how PARP-1 activation is involved in autophagy and the exact function of PARP-1-mediated autophagy under oxidative stress and DNA damage in CNE-2 cells. Recently, a study demonstrated that IR induces autophagy through a novel autophagy signaling mechanism linking PARP-1 activation to the mammalian target of rapamycin (mTOR) pathway in prostate cancer cell (20).

Questions concerning the role that autophagy plays in response to IR in $\mathrm{CNE}-2$ cells and whether it promotes cell survival or induces cell death and the methods to promote radiosensitivity by regulating autophagy are yet unanswered. Thus, it is not surprising that the bulk of research has focused on the regulation of autophagy in a variety of models $(8,11,20$ 26). Our data illustrate the importance of IR-induced autophagy, and we demonstrated that autophagic inhibition may be used as a new method to promote radiosensitivity. PARP-1-mediated autophagy plays a cytoprotective role in IR-induced CNE-2 cell death as suppression of autophagy greatly sensitizes IR-induced cell death.

\section{Materials and methods}

Cell culture and irradiation conditions. CNE-2, a human NPC cell lines, was purchased from the Cancer Hospital of Shanghai Fudan University, and was cultured in RPMI-1640 medium (HyClone, USA) supplemented with $10 \%$ fetal calf serum (Gibco, USA), penicillin (100 U/ml), streptomycin $(100 \mathrm{U} / \mathrm{ml})$ and maintained at $37^{\circ} \mathrm{C}$ in a humidified incubator with $5 \% \mathrm{CO}_{2}$. All irradiations were delivered using 6-MV $\mathrm{X}$-rays with a linear accelerator (Elekta, Sweden) with a dose rate of $220 \mathrm{cGy} / \mathrm{min}$; SSD, $100 \mathrm{~cm}$.

Reagents. Chloroquine diphosphate (CDP), an inhibitor of autophagy, was purchased from MP Biomedicals (Santa Ana, CA, USA). Rapamycin (RAPA), an inducer of cellular autophagy, was purchased from LC Laboratories (Woburn, MA, USA). PARP-1 inhibitor, 3-amino benzamide (3AB), was purchased from Sigma-Aldrich Co. (St. Louis, MO, USA), and rabbit anti-human microtubule-associated protein 1 light chain 3 (MAP1LC3B) was purchased from Sigma. Rabbit antiPARP-1 primary antibody was purchased from Cell Signaling Technology (Danvers, MA, USA), and the rabbit anti-PAR primary antibody was supplied by BD Biosciences (San Jose, CA, USA). The GAPDH primary antibody was purchased from Boster Co. (Wuhan, China), and the goat anti-mouse/ rabbit IgG secondary antibody was purchased from the KPL Co.; Annexin V-FITC apoptosis necrosis detection kit was purchased from Nanjing Kaiji Co. (Nanjing, China).
Western blotting. CNE-2 cells were washed with ice-cold PBS twice and lysed at $4^{\circ} \mathrm{C}$. The lysates were centrifuged with $12,000 \mathrm{rpm}$ at $4^{\circ} \mathrm{C}$ for $30 \mathrm{~min}$, at a centrifugal acceleration of $18,500 \mathrm{x}$ g. Protein content in the supernatants was determined using the BCA Protein Assay kit (Beyotime, Nanjing, China). Equal amounts of protein $(25 \mu \mathrm{g})$ were submitted to a $15 \%$ sodium dodecyl sulfate-polyacrylamide gel and then electrotransferred onto PVDF membranes (Merck Millipore, Billerica, MA, USA). After blocking for $2 \mathrm{~h}$, the membranes were incubated with appropriate antibodies overnight: antiLC3B $(1: 3,000)$, anti-PARP-1 $(1: 1,000)$, anti-PAR $(1: 1,500)$ and anti-GAPDH (1:800). After washing and incubating with fluorescently labeled goat anti-mouse/rabbit IgG secondary antibody $(1: 5,000)$, the fluorescence intensity was detected using the Odyssey Infrared Imaging System (LI-COR Biosciences, Lincoln, NE, USA).

Autophagosome detection. Transmission electron microscopy (TEM) (H-600 IV; Hitachi, Tokyo, Japan) was utilized for analyzing the ultrastructural images of autophagosomes and autolysosomes. CNE-2 cells were harvested by trypsinization, washed twice with PBS, and fixed with ice-cold glutaraldehyde (3\% in $0.1 \mathrm{M}$ cacodylate buffer, $\mathrm{pH} 7.4$ ) for $24 \mathrm{~h}$. The cells were post-fixed in $\mathrm{OsO}_{4}$ and dehydrated in a graded series of 70 to $100 \%$ acetone and then embedded in Epon 812. One micrometer thin sections were cut, double stained by uranium tetraacetate and lead citrate trihydrate, and viewed using TEM with a scanning attachment.

Flow cytometry. The samples were washed with phosphatebuffered saline (PBS) twice and centrifuged at 1,500 rpm for $5 \mathrm{~min}$, at a centrifugal acceleration of $1,800 \mathrm{x} \mathrm{g}$. The cells were suspended in $500 \mu \mathrm{l}$ of binding buffer [Annexin V-fluorescein isothiocyanate (FITC) kit; Kaiji, Nanjing, China], containing $5 \mu \mathrm{l}$ of Annexin V-FITC and $5 \mu \mathrm{l}$ of PI for determination of phosphatidylserine exposure on the outer plasma membrane. After incubation for 5-15 $\mathrm{min}$ at room temperature in a lightprotected area, the samples were quantified by flow cytometry (BD FACSCalibur, San Jose, CA, USA).

Assessment of cell viability using MTT assay. Cells were plated into 96 -well plates $\left(1 \times 10^{3}\right.$ cells/well, $200 \mu 1$ cell suspension/well) and cultured overnight to allow for cell attachment. After irradiation $(0,24,48$ and $72 \mathrm{~h}$ ) with $6 \mathrm{~Gy}$ X-rays, $20 \mu \mathrm{l}$ of MTT $(5 \mathrm{~g} / \mathrm{l})$ was added into each well. The cells were then incubated at $37^{\circ} \mathrm{C}$ for $4 \mathrm{~h}$, the supernatant was removed and $200 \mu \mathrm{l}$ DMSO was added. When the blue crystals were dissolved, a 96-well multiscanner autoreader (Bio-Rad M550; Bio-Rad, Hercules, CA, USA) was used to measure the absorbance value at $490 \mathrm{~nm}$ for each well. The survival rate was calculated as follows: (OD values of the experimental samples/ OD values of the control) $\mathrm{x} 100 \%$.

Clonogenic survival assay. CNE-2 cells were enzymatically dissociated with trypsin and seeded at 200, 200, 400, 600, $1,000,5,000$ and 10,000 cells/well. The cells were then irradiated ( $2 \mathrm{ml}$ of the cell suspension/well) at room temperature with $6 \mathrm{MV} \mathrm{X}$-rays with an Elekta linear accelerator with a dose rate of $220 \mathrm{cGy} / \mathrm{min}$, accordingly, with the exposure dose corresponding to $0,1,2,4,6,8$ and 10 Gy. Fourteen 
A

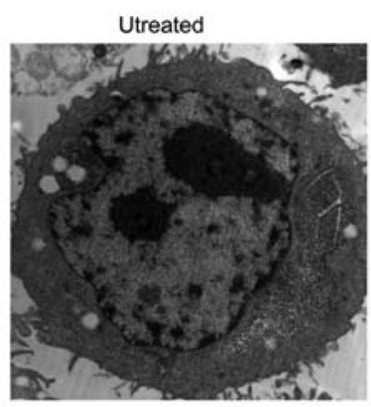

IR + CDP

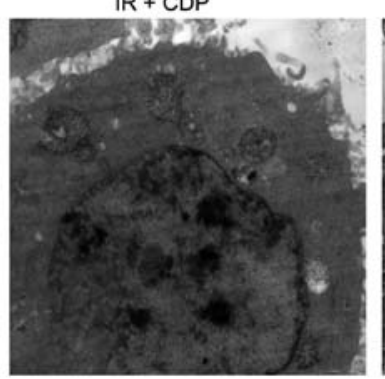

B

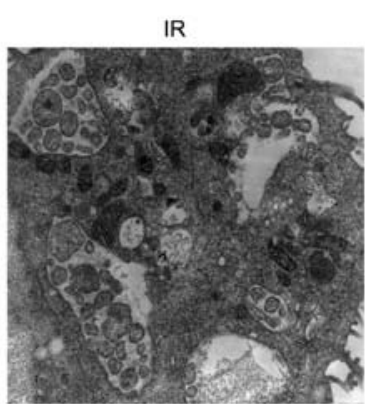

RAPA

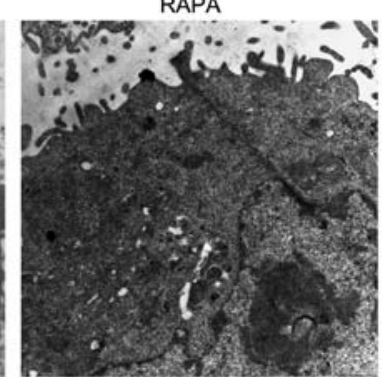

CDP

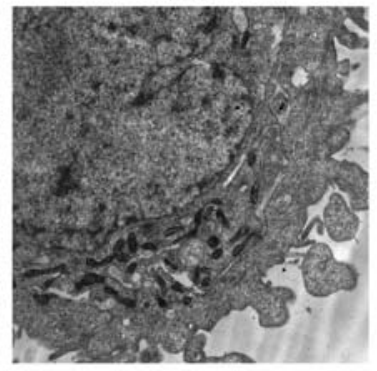

IR + RAPA

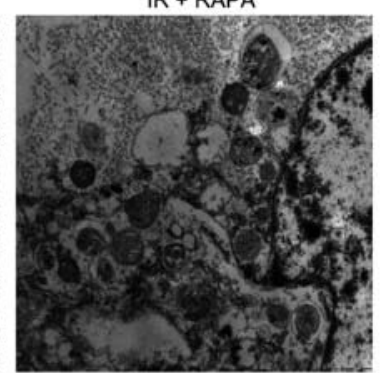

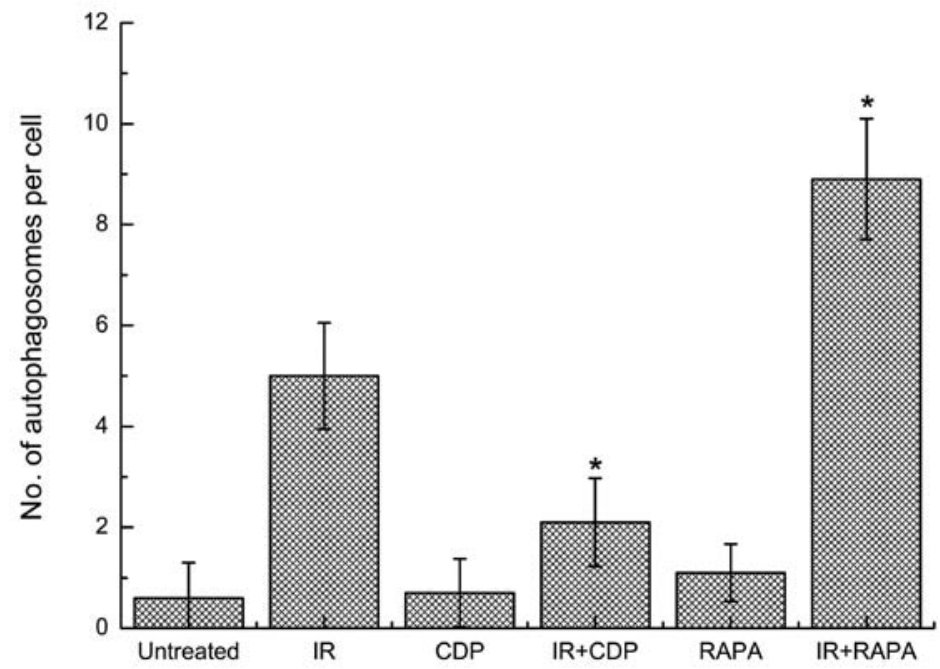

Figure 1. Detection of autophagosomes in CNE-2 cells. (A) TEM photomicrographs showed normal nuclear and mitochondrial morphologies in untreated cells and cells treated with CDP or RAPA alone. The numbers of autophagic vacuoles were obviously increased $24 \mathrm{~h}$ following IR treatment. The number of autophagosomes was decreased in cells treated with IR combined with $40 \mu \mathrm{M}$ CDP, and increased in cells treated with IR combined with $20 \mathrm{nM}$ RAPA. (B) The numbers of autophagosomes per cell are shown (means $\pm \mathrm{SD}$ ). ${ }^{*} \mathrm{P}<0.05 \mathrm{vs}$. IR alone. IR, ionizing radiation; TEM, transmission electron microscopy; CDP, chloroquine diphosphate; RAPA, rapamycin.

days later, cells were fixed with $75 \%$ ethanol and stained with Giemsa, and colonies containing $>50$ cells were counted. The surviving fraction was calculated as: Survival fraction $(\mathrm{SF})=$ experimental group colony forming efficiency/control group colony forming efficiency; Colony formation efficiency (PE) $=$ the number of colonies/plant cell number. Experiments were conducted in triplicate. Survival curves were fitted using the linear-quadratic model $\left(y=\exp \left(-\left(\alpha^{*} x+\beta^{*} x^{2}\right)\right)\right)$ using GraphPad Prism 5.0 (GraphPad Software Inc., La Jolla, CA, USA).

Statistical analysis. Statistical data are presented as means \pm standard deviation (SD). All data were subjected to analysis of variance (ANOVA) with significant differences among means identified by LSD multiple range tests using SPSS 16.0 (SPSS, Inc., Chicago, IL, USA). The criterion for statistical significance was taken as $\mathrm{P}<0.05$. At least three independent experiments were performed.

\section{Results}

Irradiation-induced accumulation of autophagosomes. Intracellular autophagosomes were observed by TEM in CNE-2 cells after IR. CDP is widely used as an inhibitor of autophagy and RAPA is widely used as an inductor of autophagy. We, therefore, examined whether CDP or RAPA had effects on the autophagy in CNE-2 cells. As shown in Fig. 1, subcellular structure analysis revealed typical morphological features of autophagy in CNE-2 cells $24 \mathrm{~h}$ after treated with IR. Autophagosomes decreased in the CNE-2 cells treated with 10-Gy irradiation combined with $40 \mu \mathrm{M} \mathrm{CDP}$, but increased in cells treated with 10-Gy irradiation combined with $20 \mathrm{nM}$ RAPA. However, in the untreated cells or cells treated with CDP or RAPA alone for $24 \mathrm{~h}$, normal nuclei surrounded by cytoplasm with normal appearing mitochondria were presented, and only occasional autophagosomes were observed. 
A

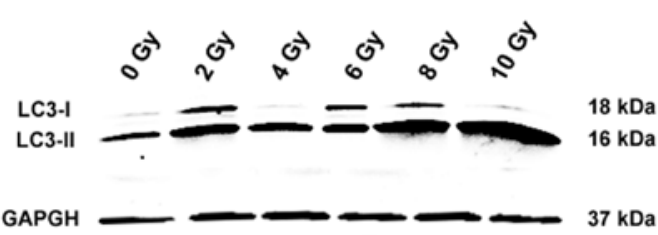

B

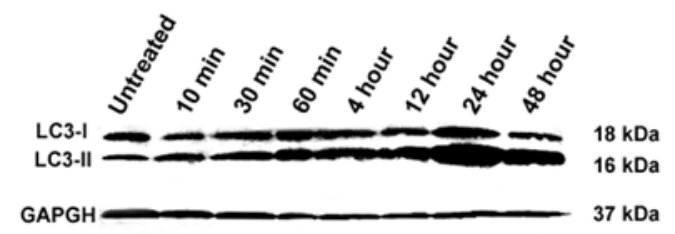

C

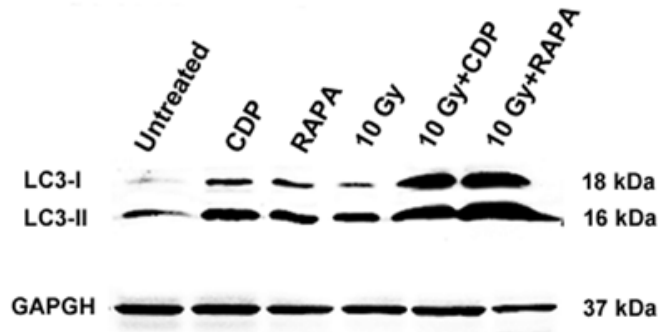

D

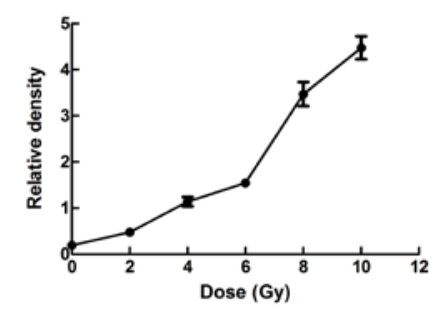

E

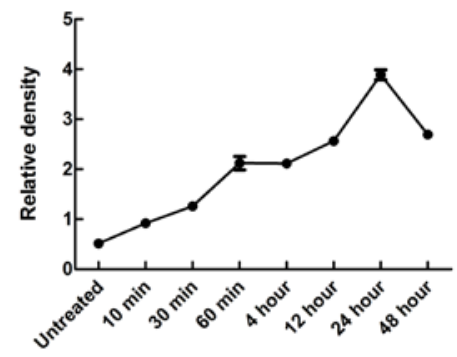

F

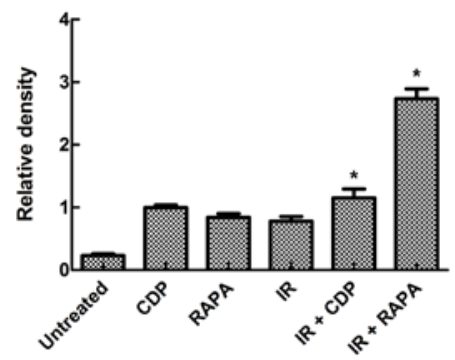

Figure 2. Detection of LC3 levels in CNE-2 cells. (A and D) The LC3-II protein level shows a dose-dependent trend induced by irradiation in CNE-2 cells. (B and E) The LC3-II protein level shows a time-dependent trend induced by irradiation in CNE-2 cells. (C and F) At $48 \mathrm{~h}$ after exposure to single or combined treatments, the ratios of LC3-I and LC3-II to GAPDH levels in each experiment were determined. The values are expressed as the means \pm SD. The differences in LC3-II expression levels in each group were statistically significant ( $\mathrm{F}=231.68, \mathrm{P}<0.01,10 \mathrm{~Gy}+\mathrm{CDP}$ or $10 \mathrm{~Gy}+\mathrm{RAPA}$ compared with the untreated group). ${ }^{*} \mathrm{P}<0.01$ vs. untreated group. CDP, chloroquine diphosphate; RAPA, rapamycin.

LC3-II expression level in CNE-2 cells treated with CDP or $R A P A$ combined with IR. To further determine the effect of IR on autophagy, the conversion of cytosolic LC3-I to LC3-II was examined in the CNE-2 cells. LC3-II protein level demonstrated a slight dose- and time-dependent increase induced by IR in the CNE-2 cells (Fig. 2A and B). LC3-II levels were correlated with the extent of autophagosome formation. Western blot assays showed that the levels of LC3-II were strongly elevated in the 10 Gy group and at $48 \mathrm{~h}$ following IR; a similar phenomenon was observed in CNE-2 cells. Significant differences in the expression level of LC3-II were found between the different experimental groups $(\mathrm{F}=231.68$, $\mathrm{P}<0.01)$. In the RAPA group, the LC3-II level was increased, and in the CDP group the LC3-II level was also increased, indicating that RAPA-induced autophagy occurred. CDP inhibits the phenomenon of autophagy. CDP destroys the structure and function of the lysosomal to inhibit autophagy, resulting in autophagic lysosomal aggregation. LC3-II was not effectively degraded, thus the LC3 expression level was upregulated in the CDP-treated group, confirming that CDP inhibits autophagy. Compared with the untreated group, the level of LC3-II was significantly higher in the RAPA + 10 Gy group $(\mathrm{P}<0.01)$ and CDP group $(\mathrm{P}<0.01)$ (Fig. $2 \mathrm{C})$.

PARP-1 activation contributes to irradiation-induced cell autophagy. PARP-1 is readily activated in response to DNA damage and is well associated with cell death. As shown in Fig. 3A and B, increased formation of the PAR polymer, a direct result of PARP-1 activation, was detected as early as $10 \mathrm{~min}$ after IR exposure. To verify whether PARP-1 activation contributes to the cell autophagy induced by IR, cells were treated with $10 \mathrm{~Gy}$ X-rays in the presence or absence of 3-amino benzamide (3AB), a specific PARP-1 inhibitor. Pretreatment with $3 \mathrm{AB}$ significantly inhibited IR-induced PAR formation. PAR and LC3-II both showed a certain degree of dosedependence. Changes in PAR content were noted obviously earlier than LC3-B changes, and PAR was soon degraded. This suggests that PAR may be upstream of the signaling pathways. In order to further verify if the PARP-1 signaling pathway is upstream, we used the PARP-1 chemical inhibitor $3 \mathrm{AB}$, autophagy inhibitor CDP and autophagy inducer RAPA to pretreat CNE-2 cells, and $2 \mathrm{~h}$ after $10 \mathrm{~Gy}$ IR, cells were collected and the protein was extracted, and changes in the levels of LC3-I and -II and PAR were determined. (Fig. 3C and D). The results clearly suggested that PARP-1 activation contributed to IR-induced cell autophagy. TEM examination further confirmed this finding (Fig. 4).

Autophagy or PARP-1 inhibition increases the apoptosis rate in CNE-2 cells following irradiation. IR combined with autophagy inhibitor or inducer caused CNE-2 cell apoptosis; $48 \mathrm{~h}$ after IR, the apoptosis rates of CNE-2 cells in the untreated group, 
A

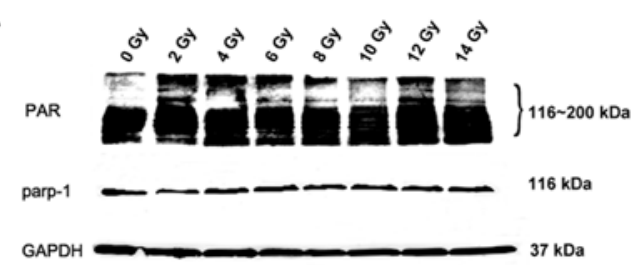

B

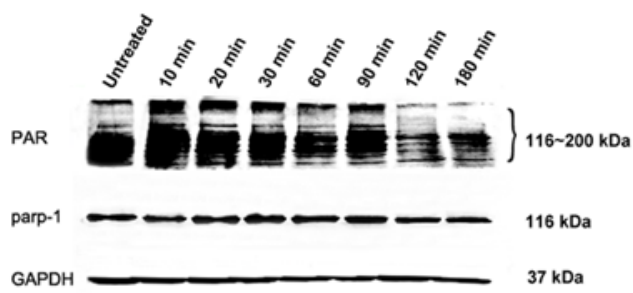

C

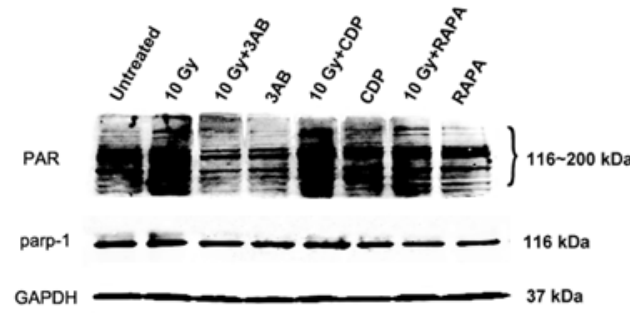

D

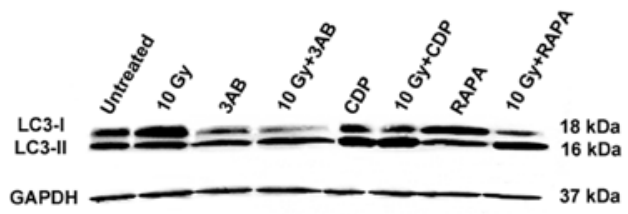

E

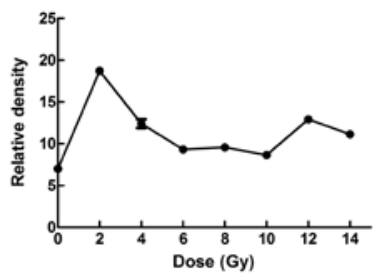

$\mathbf{F}$

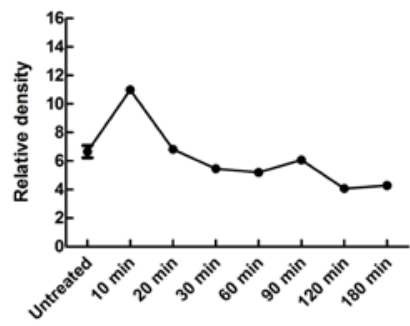

G

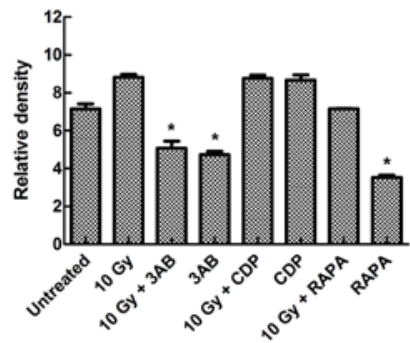

H

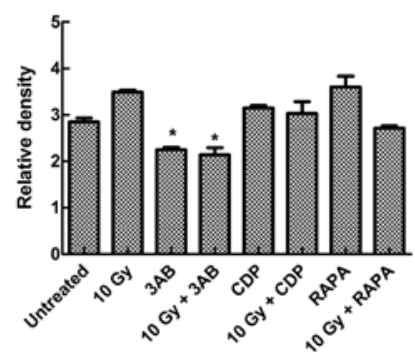

Figure 3. Detection of PAR, PARP-1 and LC3 levels in CNE-2 cells. (A and E) The PAR protein level induced by irradiation showed a slight dose-dependent trend in CNE-2 cells. (B and F) Increased formation of poly(ADP-ribose) (PAR) polymer was detected in CNE-2 cells as early as 10 min after irradiation exposure, and the PAR protein level decreased to normal after 2 h. (C and G) Effect of 3AB on IR-induced PAR activation and cell autophagy 15 min after exposure to single or combined treatments. Cells were treated with IR with or without a 2-h pretreatment of $3 \mathrm{AB}(10 \mathrm{mM})$. Cells were collected and subjected to western blot analysis $15 \mathrm{~min}$ after exposure. The level of PAR was quantified and the means (fold of changes over the control group) of three independent experiments are presented. The level of PAR was significantly lower in $10 \mathrm{~Gy}+3 \mathrm{AB}$ and $3 \mathrm{AB}$ groups compared with IR alone group (both $\mathrm{P}<0.01$ ), however, no significant difference was observed in either $10 \mathrm{~Gy}+\mathrm{CDP}$ or CDP group compared with the IR alone group (both P>0.05). (D and H) The levels of LC3-II in $10 \mathrm{~Gy}+3 \mathrm{AB}$ and $3 \mathrm{AB}$ groups were lower compared with the IR alone group ( $\mathrm{P}<0.01)$. CDP, chloroquine diphosphate; RAPA, rapamycin; 3AB, 3-amino benzamide; IR, ionizing radiation.

CDP group, RAPA group, 3AB group, IR group, IR + CDP group, IR + RAPA group and IR + 3AB group, were $7.71 \pm 0.90$, $8.29 \pm 1.60,9.20 \pm 1.59,9.91 \pm 0.75,26.63 \pm 3.11,31.28 \pm 1.58$, $34.19 \pm 1.15$ and $30.80 \pm 3.33 \%$, respectively. Significant differences in the apoptosis rates were observed between the groups $(\mathrm{F}=109.69, \mathrm{P}<0.01)$. The apoptosis rate was significantly higher in the CDP + IR, RAPA + IR and $3 \mathrm{AB}+$ IR group than that in the IR alone group $(\mathrm{P}<0.01)$ (Fig. 5A). At 24 or $48 \mathrm{~h}$ after IR, a similar phenomenon was observed, but at $72 \mathrm{~h}$ after IR, RAPA or $3 \mathrm{AB}$ combined with IR promoted cell survival rather than enhanced CNE-2 cell apoptosis (Fig. 5B).

Autophagy or PARP-1 inhibitor contributes to inhibition of CNE-2 cell proliferation and survival rate after irradiation. MTT assay was used to further elucidate whether the sensitivity of CNE-2 cells to IR occurred through autophagy or PARP-1 inhibition. The absorbance value indicates the proliferation and survival of cells; the higher the absorbance value, the higher the number of surviving cells. The proliferation rate of the CDP, RAPA or $3 \mathrm{AB}$ combined with 6 Gy IR groups was lower than that of the IR alone group (Fig. 6A). There were significant differences in the number of surviving cells and the survival rate between the different treatment groups $72 \mathrm{~h}$ after IR ( $\mathrm{F}=50.40$, $\mathrm{P}<0.01$ ) (Fig. 6A). The survival rates of the CDP, RAPA or $3 \mathrm{AB}$ combined with 6 Gy IR group were significantly lower than that of the IR alone group, at each time point $(\mathrm{P}<0.01)$ (Fig. 6B).

Autophagy or PARP-1 inhibitor contributes to the radiation sensitization of CNE-2 cells. As expected, the effect of CDP, RAPA and $3 \mathrm{AB}$ on the lethality of IR was ascertained by 
A

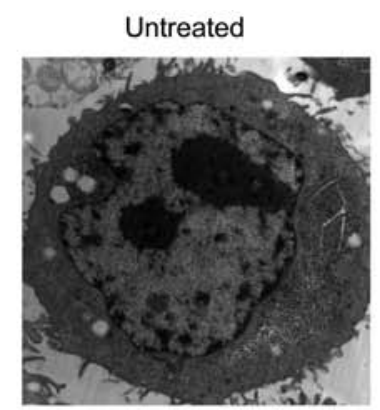

$3 A B$

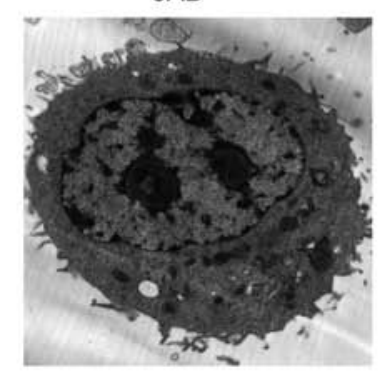

IR

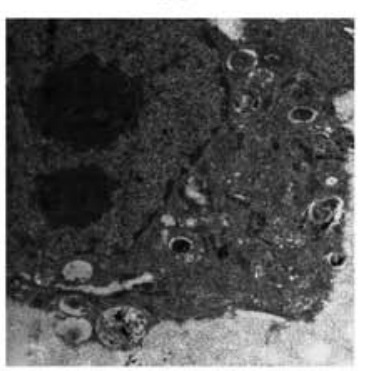

$\mathrm{IR}+3 \mathrm{AB}$

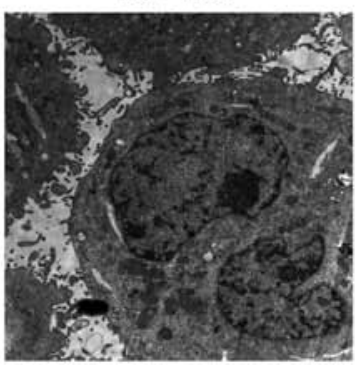

B

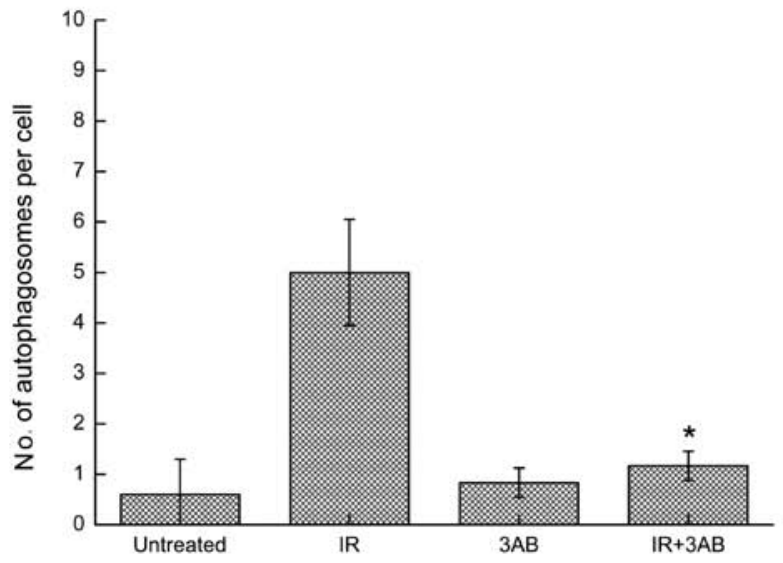

Figure 4. Detection of autophagy in CNE-2 cells. (A) TEM photomicrographs show normal nuclear and mitochondrial morphologies in the untreated cells and cells treated with IR $+3 \mathrm{AB}, 3 \mathrm{AB}$ or IR alone. (B) The numbers of autophagic vacuoles was obviously increased $24 \mathrm{~h}$ following IR treatment. The number of autophagosomes decreased in the IR combined with $10 \mathrm{mM} 3 \mathrm{AB}$ group. The numbers of autophagosomes per cell are shown (means $\pm \mathrm{SD}$ ). "P<0.05 vs. IR alone. IR, ionizing radiation; TEM, transmission electron microscopy; 3AB, 3-amino benzamide.

A
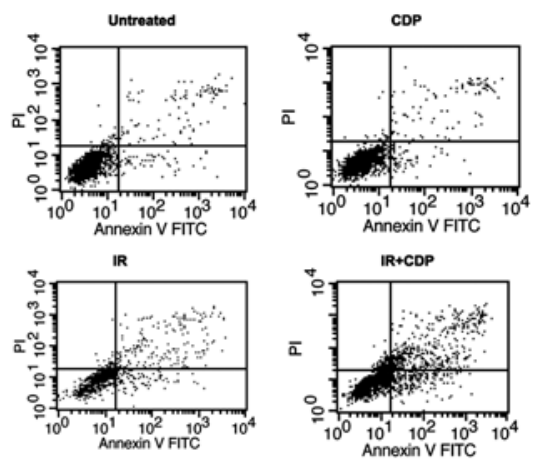

B

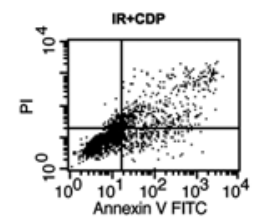

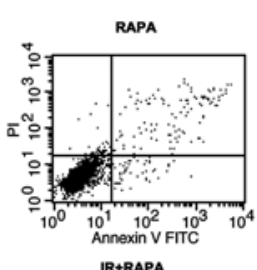
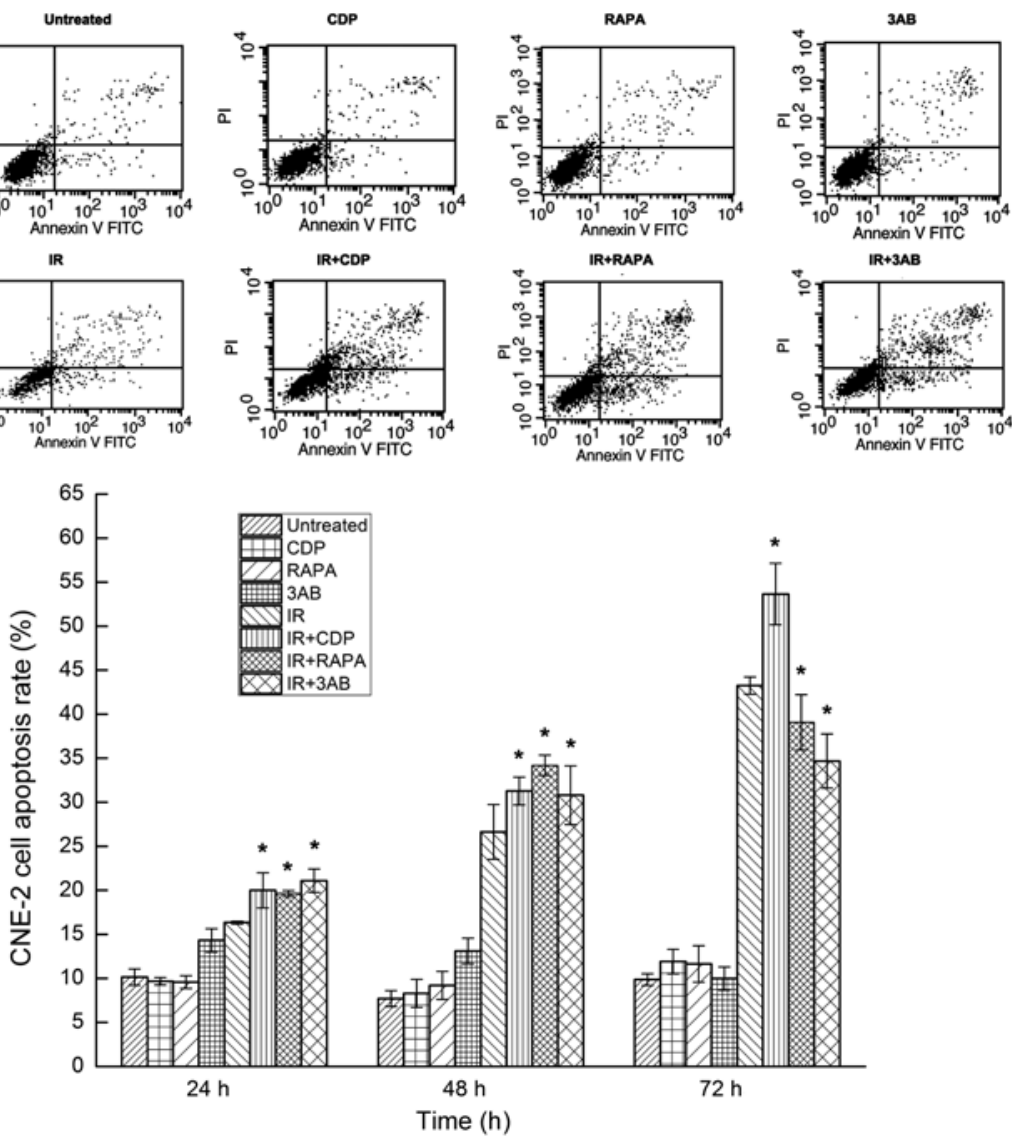

Figure 5. Analysis of CNE-2 cell apoptosis. (A) Cell apoptosis was detected by flow cytometry $48 \mathrm{~h}$ after IR. The apoptosis rate was evaluated by staining of

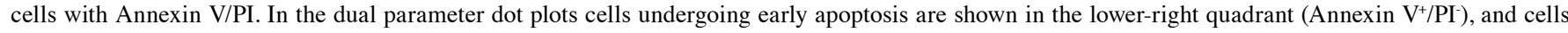
undergoing late apoptosis are shown in the upper right quadrant $\left(\right.$ Annexin $\left.\mathrm{V}^{+} / \mathrm{PI}^{+}\right)$. The apoptosis rate was higher in the IR + CDP, IR + RAPA and IR + 3AB groups, when compared with that in the IR alone group; the difference was statistically significant $(\mathrm{P}<0.05)$. (B) Cell apoptosis was detected by flow cytometry at 24, 48 and $72 \mathrm{~h}$ after IR, respectively. CDP combined with IR, RAPA combined with IR, and 3AB combined with IR all enhanced CNE-2 cell apoptosis at 24 and $48 \mathrm{~h}$ after irradiation. However, CDP combined with IR continued to enhance cell apoptosis at $72 \mathrm{~h}$ after IR while RAPA combined with IR and $3 \mathrm{AB}$ combined with IR promoted cell survival rather than enhanced cell apoptosis. ${ }^{*} \mathrm{P}<0.05$ vs. IR alone. CDP, chloroquine diphosphate; RAPA, rapamycin; 3AB, 3-amino benzamide; IR, ionizing radiation. 
A

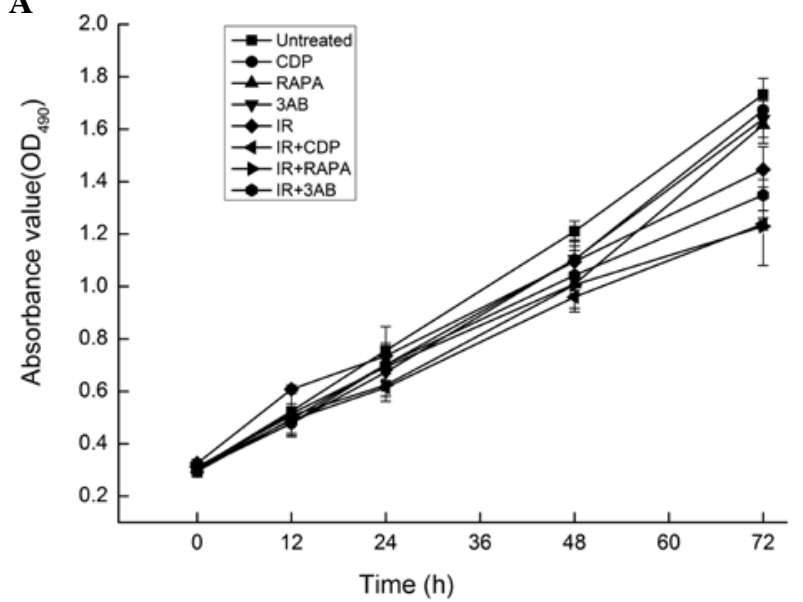

B

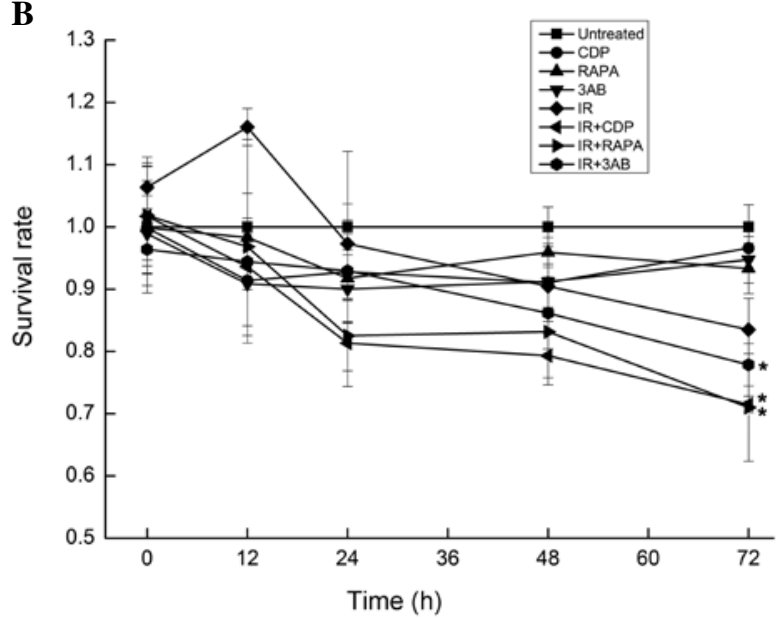

Figure 6. Effects of different treatment procedures on the viability of CNE-2 cells. (A) Cell proliferation was assessed by MTT method. (B) The survival rate was calculated as follows: (OD values of the experimental samples/OD values of the control) $x 100 \%$. Data are presented as means $\pm \mathrm{SD}$, $\mathrm{n}=3$. ${ }^{*} \mathrm{P}<0.05$, vs. IR alone. CDP, chloroquine diphosphate; RAPA, rapamycin; 3AB, 3-amino benzamide; IR, ionizing radiation.

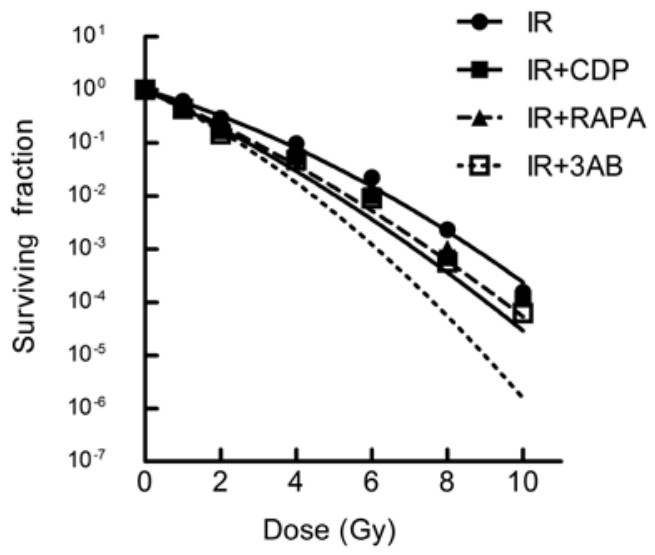

Figure 7. Cell survival curves of the CNE-2 cells. Clonogenic survival assay was performed to evaluate long-term cell survival. The cell survival curves of CNE-2 cells treated with IR alone or in combination with CDP, RAPA or $3 \mathrm{AB}$ were assessed using the linear-quadratic formulation. $\mathrm{CDP}$, chloroquine diphosphate; RAPA, rapamycin; 3AB, 3-amino benzamide; IR, ionizing radiation.

clonogenic survival assay. GraphPad Prism 5.0 Software using the linear-quadratic model was used to calculate the radiobiology parameters and fitting dose survival curve (Table I and Fig. 7). Compared with IR alone, the IR + CDP group caused increased radiosensitivity of CNE-2 cells; RAPA or $3 \mathrm{AB}$ combined with IR showed a similar role and suggests that autophagy inhibition may be an approach to enhance the lethality of radiation.

\section{Discussion}

Apoptosis is a process of programmed cell death, autophagy is a process of programmed cell survival (27). Yet, too much or too little autophagy can damage cells. In some cases, autophagy can cause cell death. Several reports in the early literature also called autophagy 'type II programmed cell death', but now it is a misnomer $(28,29)$. At present, concerning the relationship between autophagy and radiation sensitization of tumor cells,
Table I. Radiobiology parameters (the linear-quadratic model).

\begin{tabular}{lccr}
\hline Group & $\alpha$ & $\beta$ & $\alpha / \beta$ \\
\hline IR & $0.496 \pm 0.013$ & $0.034 \pm 0.005$ & $14.898 \pm 2.932$ \\
IR + CDP & $0.770 \pm 0.017^{\mathrm{a}}$ & $0.029 \pm 0.014$ & $34.028 \pm 22.440$ \\
IR + RAPA & $0.706 \pm 0.026^{\mathrm{a}}$ & $0.029 \pm 0.015$ & $29.673 \pm 16.713$ \\
IR + 3AB & $0.785 \pm 0.033^{\mathrm{a}}$ & $0.057 \pm 0.023$ & $15.769 \pm 7.553$ \\
F-value & 97.631 & 2.247 & 1.333 \\
P-value & 0.000 & 0.160 & 0.330 \\
\hline
\end{tabular}

Each value is expressed as the means $\pm \mathrm{SD}$. ${ }^{\mathrm{a}} \mathrm{P}<0.01$, vs. IR alone. $\mathrm{CDP}$, chloroquine diphosphate; RAPA, rapamycin; $3 \mathrm{AB}$, 3-amino benzamide; IR, ionizing radiation.

several scholars believe that inhibition of autophagy of tumor cells can improve the effect of radiotherapy $(11,21,23)$. Chen et al (21) found that autophagy inhibitor 3-methyladenine (3-MA) combined with radiation increased the apoptosis of esophageal squamous carcinoma cells. Restraining autophagy increased the cytotoxicity of radiotherapy in esophageal carcinoma cells and arrested the cell cycle in the $G_{2} / M$ phase, increasing the sensitivity of radiotherapy. Ito et al (23) found that autophagy inhibitors, 3-MA and bafilomycin A1, increased the sensitivity of malignant glioma U373-MG cells. Meanwhile, radiation caused increased DNA double chain ruptures after inhibition of autophagy. Thus, autophagy inhibitors may become a type of new radiotherapy sensitization agents for malignant glioma. Several researchers also suggest that inducing autophagy improves the effect of radiotherapy on tumors $(20,22)$. Cao et al (20) found that mTOR inhibitors, rapamycin and RAD001, inhibit mTOR function and induce autophagy, consquently improving the radiotherapy effect in breast cancer cells.

The present study demonstrated that radiation causes autophagy, and LC3-II protein levels were increased in a dose- and time-dependent manner induced by irradiation in 
CNE-2 cells. LC3-II is currently the only protein identified which is present in autophagic bodies and autophagy-lysosome membrane (30). Our research also detected autophagy body formation and the quantity in nasopharyngeal carcinoma cells by TEM which is the gold standard of autophagy detection, which further corroborated the test results of the western blot analysis.

Autophagy inhibitor CDP damages the structure and function of lysosomes to inhibit autophagy. It causes autophagy-lysosome to gather together and the degradation of LC3-II is reduced. RAPA is an autophagy inducer and causes Atg13 dephosphorylated and Atg1 activation by inhibiting mTOR activity to induce autophagy (24). In this study, CDP inhibited CNE-2 cell autophagy and RAPA induced CNE-2 cell autophagy caused by irradiation (31). It is important to note that CDP, by destroying the lysosome structure and function, inhibits autophagy, causes autophagy-lysosome together, and reduces LC3-II degradation. LC3-II failed to undergo effective decomposition, thus in the CDP-treated group, LC3-II expression level was increased, which confirmed that CDP inhibits autophagy; the characteristic of CDP is different from the autophagic inhibitor 3-MA (21).

Flow cytometry detected the apoptosis rate of the different groups at 24, 48 and $72 \mathrm{~h}$ after radiation. Our study showed that the apoptosis rate of the CDP + IR group significantly increased at the three time points compared with the IR alone group, which indicated that autophagy inhibitors can improve the sensitivity of the early response of nasopharyngeal carcinoma cells to IR. Although the autophagy inducer RAPA combined with IR significantly decreased the apoptosis rate at $72 \mathrm{~h}$ after radiation compared with the IR alone group, it significantly increased rather than decreased the apoptosis rate at 24 or $48 \mathrm{~h}$ after radiation. The results concerning the effect of RAPA were contradictory, and the reasons may be as follows: i) the characteristics of the RAPA drug itself, which was used widely as an antifungal drug; ii) physiological autophagy is a dynamic balance; a dysregulated balance may cause damage; 10 Gy IR combined with RAPA have synergy, and excessive autophagy may be also a type of cell death consequently leading to an increase in the apoptosis rate. Autophagy is a double-edged sword. Autophagy caused by IR inhibits the cell death process and plays a protective role. Thus, inhibiting autophagy can increase the radiation sensitization of CNE-2 cells. However, excessive autophagy may also cause cell death $(6,7,12,13)$. According to the radiation biology, the surviving cells after radiation require concern. Only those cells that lose their proliferation capability and are unable to form effective clone cells are unable to cause cancer recurrence. We used a quadratic linear model to construct dose survival curves and calculate the radiation biology parameters. The results suggest that autophagy inhibitors significantly increased radiation sensitization, autophagy inducers showed a similar effect. The proliferation of the cells treated with an autophagy inhibitor combined with IR decreased.

In the present study, we identifed a novel function for PARP-1 in mediating IR-induced autophagy and such autophagy plays a pro-survival function in IR-induced cell death. Although at the $72-\mathrm{h}$ time point, $3 \mathrm{AB}$ promoted $\mathrm{CNE}-2$ cell survival rather than enhanced cell apoptosis, a clonogenic survival assay was performed to evaluate long-term cell survival. The result showed that the PARP-1 inhibitor contributed to radiation sensitization of CNE-2 cells (16). It appears that PARP-1 is able to elicit dual pathways with opposite functions in response to oxidative stress (31), as illustrated in Fig. 5B. The decision of cell life or death is dependent on the balance between apoptosis and autophagy mediated by these two distinct pathways. Oxidative DNA damage includes modifications to bases and the sugar phosphates, as well as single- or double-strand DNA breaks. Such damage leads to PARP-1 activation, suppression of ATP production and finally cell death. Furthermore, overactivation of PARP-1 produces large quantities of PAR polymers, leading to autophagy, and finally cell survival.

In summary, we demonstrated a novel function of PARP-1 in the regulation of IR-induced autophagy through the mTOR signaling pathway, and such autophagy serves as a cell survival mechanism against IR-mediated cell death. Autophagy inhibitors and PARP-1 inhibitors contributed to the radiation sensitization of CNE-2 cells. This suggests that CDP or $3 \mathrm{AB}$ may be used as adjuvant treatment for nasopharyngeal carcinoma. Further animal experiments and clinical tests are warranted to verify our findings.

\section{Acknowledgements}

The authors thank Zhangyu Zou from the Department of Neurology of Peking Union Medical College Hospital, Chinese Academy of Medical Sciences and Peking Union Medical College, for the valuable discussions. The study was supported by the NSFC (Natural Science Foundation of China) (81160285) and the Guangxi Natural Science Foundation (2010gxnsfa013240).

\section{References}

1. Wei WI and Sham JST: Nasopharyngeal carcinoma. Lancet 365: 2041-2054, 2005

2. Feng XP, Yi H, Li MY, et al: Identification of biomarkers for predicting nasopharyngeal carcinoma response to radiotherapy by proteomics. Cancer Res 70: 3450-3462, 2010.

3. Guo Y, Zhu XD, Qu S, et al: Identification of genes involved in radioresistance of nasopharyngeal carcinoma by integrating gene ontology and protein-protein interaction networks. Int $\mathrm{J}$ Oncol 40: 85-92, 2012.

4. Mizushima N: Autophagy: process and function. Genes Dev 21: 2861-2873, 2007

5. Mizushima N, Levine B, Cuervo AM and Klionsky DJ: Autophagy fights disease through cellular self-digestion. Nature 451: 1069-1075, 2008.

6. Degenhardt K, Mathew R, Beaudoin B, et al: Autophagy promotes tumor cell survival and restricts necrosis, inflammation, and tumorigenesis. Cancer Cell 10: 51-64, 2006.

7. Shintani T and Klionsky DJ: Autophagy in health and disease: a double-edged sword. Science 306: 990-995, 2004.

8. Komatsu M, Waguri S, Chiba T, et al: Loss of autophagy in the central nervous system causes neurodegeneration in mice. Nature 441: 880-884, 2006

9. Hara T, Nakamura K, Matsui M, et al: Suppression of basal autophagy in neural cells causes neurodegenerative disease in mice. Nature 441: 885-889, 2006.

10. Dalby KN, Tekedereli I, Lopez-Berestein G and Ozpolat B: Targeting the prodeath and prosurvival functions of autophagy as novel therapeutic strategies in cancer. Autophagy 6: 322-329, 2010.

11. Apel A, Herr I, Schwarz H, Rodemann HP and Mayer A: Blocked autophagy sensitizes resistant carcinoma cells to radiation therapy. Cancer Res 68: 1485-1494, 2008.

12. Marx J: Autophagy: is it cancer's friend or foe? Science 312: 1160-1161, 2006. 
13. Wu WK, Coffelt SB, Cho $\mathrm{CH}$, et al: The autophagic paradox in cancer therapy. Oncogene 31: 939-953, 2012.

14. Schreiber V, Dantzer F, Ame JC and de Murcia G: Poly(ADPribose): novel functions for an old molecule. Nat Rev Mol Cell Biol 7: 517-528, 2006.

15. Yu SW, Wang H, Poitras MF, et al: Mediation of poly(ADPribose) polymerase-1-dependent cell death by apoptosis-inducing factor. Science 297: 259-263, 2002.

16. Jagtap P and Szabo C: Poly(ADP-ribose) polymerase and the therapeutic effects of its inhibitors. Nat Rev Drug Discov 4 421-440, 2005.

17. Ha HC and Snyder SH: Poly(ADP-ribose) polymerase is a mediator of necrotic cell death by ATP depletion. Proc Natl Acad Sci USA 96: 13978-13982, 1999.

18. Albert JM, Cao C, Kim KW, et al: Inhibition of poly (ADP-ribose) polymerase enhances cell death and improves tumor growth delay in irradiated lung cancer models. Clin Cancer Res 13 3033-3042, 2007.

19. Hagan MP, Yacoub A and Dent P: Radiation-induced PARP activation is enhanced through EGFR-ERK signaling. J Cell Biochem 101: 1384-1393, 2007.

20. Cao C, Subhawong T, Albert JM, et al: Inhibition of mammalian target of rapamycin or apoptotic pathway induces autophagy and radiosensitizes PTEN null prostate cancer cells. Cancer Res 66 : 10040-10047, 2006

21. Chen YS, Song HX, Lu Y, et al: Autophagy inhibition contributes to radiation sensitization of esophageal squamous carcinoma cells. Dis Esophagus 24: 437-443, 2011.

22. Lomonaco SL, Finniss S, Xiang CL, et al: The induction of autophagy by gamma-radiation contributes to the radioresistance of glioma stem cells. Int J Cancer 125: 717-722, 2009.
23. Ito H, Daido S, Kanzawa T, Kondo S and Kondo Y: Radiationinduced autophagy is associated with LC3 and its inhibition sensitizes malignant glioma cells. Int J Oncol 26: 1401-1410, 2005.

24. Paglin S, Lee NY, Nakar C, et al: Rapamycin-sensitive pathway regulates mitochondrial membrane potential, autophagy, and survival in irradiated MCF-7 cells. Cancer Res 65: 11061-11070, 2005.

25. Rieber M and Rieber MS: Sensitization to radiation-induced DNA damage accelerates loss of bcl-2 and increases apoptosis and autophagy. Cancer Biol Ther 7: 1561-1566, 2008.

26. Peng PL, Kuo WH, Tseng HC and Chou FP: Synergistic tumorkilling effect of radiation and berberine combined treatment in lung cancer: the contribution of autophagic cell death. Int J Radiat Oncol Biol Phys 70: 529-542, 2008.

27. Maiuri MC, Zalckvar E, Kimchi A and Kroemer G: Self-eating and self-killing: crosstalk between autophagy and apoptosis. Nat Rev Mol Cell Biol 8: 741-752, 2007.

28. Kroemer G and Levine B: Autophagic cell death: the story of a misnomer. Nat Rev Mol Cell Biol 9: 1004-1010, 2008.

29. Levine B and Yuan JY: Autophagy in cell death: an innocent convict? J Clin Invest 115: 2679-2688, 2005.

30. Galluzzi L, Aaronson SA, Abrams J, et al: Guidelines for the use and interpretation of assays for monitoring cell death in higher eukaryotes. Cell Death Differ 16: 1093-1107, 2009.

31. Huang Q and Shen HM: To die or to live: the dual role of poly(ADP-ribose) polymerase-1 in autophagy and necrosis under oxidative stress and DNA damage. Autophagy 5: 273-276, 2009. 